\title{
Estudio bibliométrico de publicaciones científicas que utilizan pruebas de reconocimiento de emociones faciales
}

\author{
Graciela Jeanneret, Astrid Oña, Pamela Rego, Marcelo Vaiman y Germán Pereno* \\ Laboratorio de Psicología Cognitiva, Facultad de Psicología, Universidad Nacional de Córdoba, Argentina
}

\begin{abstract}
Resumen: El trabajo se enmarca dentro de la perspectiva evolucionista, la cual sostiene la existencia de emociones universales, enfatizando el estudio de su expresión facial y su reconocimiento. Si bien la creación del sistema FACS en 1978 por Ekman impulsó la construcción de instrumentos de evaluación del reconocimiento de emociones faciales, actualmente no existen pruebas locales que midan dicha habilidad.

El objetivo de este estudio fue la realización de un análisis bibliométrico de investigaciones científicas publicadas entre 1976 y agosto de 2011 que utilizan pruebas de reconocimiento de emociones faciales. Este proceso de sistematización de los documentos brinda visibilidad sobre el estado actual de la ciencia en relación a dichas pruebas.

Se analizaron los indicadores de productividad, colaboración, índice h y análisis de materia. Éstos evidenciaron un crecimiento sistemático cada vež más pronunciado en la utilización de investigaciones en el reconocimiento de emociones faciales. Estas utilizan fotografías tales como el POFA y el JACFEE en población adulta de ambos sexos, adquiriendo relevancia el estudio de patologías, principalmente la esquizofrenia.

Estos resultados, a su vez, podrán utilizarse como fundamento para posteriores proyectos de investigación que aporten al progreso de la ciencia.

Palabras Claves: Prueba de reconocimiento de Emociones Faciales; estudio bibliométrico; perspectiva Evolucionista; Ekman.
\end{abstract}

\section{Introducción}

Entre las diferentes orientaciones abocadas al estudio de las emociones se destacó, por sus importantes aportes, la perspectiva evolucionista (Weisfeld \& LaFreniere, 2012). Ésta situó el énfasis en la capacidad para expresar y reconocer emociones en rostros y tomó como base los postulados de la tesis darwiniana sobre la existencia de emociones universales. El principal exponente de esta línea teórica es Paul Ekman, quien llevó a cabo sus investigaciones transculturales a partir de la década del 60.

Ekman realizó sus primeros estudios en cinco culturas literatas (Ekman, Sorenson y Friesen, 1969; Ekman, 1972), que consistían en mostrar fotografías a individuos de cinco países diferentes para que éstos identificasen cuál era la expresión emocional representada en la imagen. Los resultados de estas investigaciones mostraron que la mayoría de los sujetos de cada cultura acordaban en su respuesta, sugiriendo ello que las expresiones emocionales podrían ser universales (Ekman, 2003).

Siguiendo la misma línea de investigación, en su estudio sobre nueve culturas literatas, Izard (1971) agregó evidencias a favor de los hallazgos reportados por Ekman, es decir, que existían acuerdos entre las culturas en la interpretación de las

\section{* Dirección para correspondencia [Correspondence address]}

Germán Pereno. Facultad de Psicología. Universidad Nacional de Córdoba. Enfermera Gordillo esquina Enrique Barros, Ciudad Universitaria. Córdoba (Argentina).

E-mail: gpereno@psyche.unc.edu.ar
Title: A bibliometric study of the scientific publications that use facial emotion recognition tests.

Abstract: This work is in line with the evolutionary theory, which supports the existence of basic and universal emotions, stress the study of his facial expression and his recognition. Though the development of FACS in 1978 for Ekman motivated the creation of facial emotion recognition test, there is not in Argentine test that measure the above mentioned ability.

The aim of this study was carry out a bibliometric study of the scientific publications that use facial emotion recognition tests published between 1976 and August, 2011. This process of systematizing offers visibility on the current condition of the science in relation to the above mentioned tests, constituting the first step for the development of a local test.

In effect, there were analyzed the productivity, scientific collaboration, hindex and matter analysis indicators. These demonstrated a systematic growth increasingly in researches in facial emotions recognition. These studies use photographs as the POFA and the JACFEE in adult population of both male and female sexes, becomes more relevant the study of pathologies, mainly the schizophrenia.

These results, in turn, will be able to be in use as base for successive research projects that contribute to the progress of the science.

Key words: Facial emotions recognition test; bibliometric study; evolutionary theory; Ekman.

expresiones faciales de los observadores, aún cuando se les permitió elegir sus propias palabras para describir las emociones (Quinn et al., 2011). De esta manera, ambos autores por separado, comprobaron la existencia en diversas culturas de al menos seis expresiones faciales universales, correspondientes a seis de las emociones que tanto Darwin como Tomkins habían considerado como innatas: alegría, ira, miedo, sorpresa, asco y tristeza (Iglesias, Loeches y Serrano, 1989).

Ekman, Izard y Plutchik consideraron que la cara constituye el lugar principal donde se asientan las emociones y defendieron que el valor adaptativo de las expresiones faciales radica en que transmiten de un modo confiable el estado emocional del emisor y su tendencia conductual, teniendo una influencia directa en el establecimiento y regulación del contacto social (Iglesias et al., 1989).

La expresión de las emociones constituye una capacidad de suma importancia para comunicar sensaciones y sentimientos, como así también, para transmitir a los demás distintos indicadores del estado emocional y proveer señales que permitan a los semejantes responder de manera adecuada ante el propio estado anímico (Ten Brinke, Macdonald, Porter, \& O'Connor, 2012). Esto se debe a que el lenguaje emocional es el más primitivo, tanto en sentido filogenético como ontogenético, siendo un procesamiento emocional correcto fundamental para un desarrollo emocional normal (Herba y Phillips, 2004). Cabe destacar que, el hecho de considerar que las expresiones faciales de emociones son buenos indicadores conductuales de la emoción, se debe quizás, entre otras razones, a su importancia para la comunicación y la 
regulación de las interacciones sociales (Preuschoft, 2000; Wong, Beidel, Sarver, \& Sims, 2012).

Un apartado especial merece los nuevos desarrollos que surgieron luego de considerar la importancia del lenguaje y la inteligencia emocional (Porter, ten Brinke, \& Wallace, 2012). Éste fue utilizado, entre otros, por la Psicología Forense, la cual incluye la toma de decisiones jurídicas, el comportamiento criminal, la victimología, la detección de mentiras, los trastornos mentales y la delincuencia, la personalidad y los crímenes. Se destacan aquí los trabajos del psicólogo forense Stephen Porter, el cual desde una perspectiva evolucionista ha estudiado principalmente las expresiones emocionales involuntarias que normalmente se producen solo en la parte superior o inferior del rostro (Porter, ten Brinke, Baker, \& Wallace, 2012).

Dado que las expresiones faciales son manifestaciones observables del estado emocional del individuo, se tornó más factible su abordaje por medio de métodos sistemáticos que permitieran realizar evaluaciones y mediciones precisas. En este sentido, la perspectiva evolucionista se destacó por desarrollar diversos sistemas de codificación que evalúan las distintas expresiones emocionales que se manifiestan en el rostro, siendo el más reconocido el FACS (Facial Action Coding System; Ekman y Friesen, 1978). Dicho sistema fue diseñado para la detección de cambios en las expresiones faciales, ya sean notorias o microexpresiones (aquellas que tienen lugar en milésimas de segundos y que pueden ocurrir de forma individual o conjuntamente con otras), sosteniendo que las expresiones faciales específicas corresponden a expresiones faciales prototípicas. Incluso en la actualidad se han desarrollado programas computacionales automáticos para superar las limitaciones del sistema FACS (Hamm, Kohler, Gur, \& Verma, 2011).

El FACS fue utilizado por Ekman y Friesen (1976) para el desarrollo y la validación del Pictures of Facial Affect (POFA). El POFA está constituido por 110 fotografías de sujetos expresando algunas de las emociones básicas más la categoría neutral, las cuales han sido utilizadas en una amplia gama de estudios transculturales, de neuroimágen y en investigación conductual (Matsumoto, Keltner, Shiota, O'Sullivan, y Frank, 2008). En la actualidad, cobraron un interés aún mayor en el campo de la Neurociencia Cognitiva, específicamente en el estudio de las conductas sociales o la Cognición Social (Adolphs, 2003), relacionando para una mejor comprensión del cerebro social perspectivas evolucionistas y psicológicas (Sutcliffe, Dunbar, Binder, \& Arrow, 2012).

Actualmente, existe una gran cantidad y variabilidad en cuanto a las pruebas utilizadas para evaluar la capacidad de reconocimiento de emociones, a saber: 1) conjunto de imágenes de personas expresando las emociones básicas descriptas por Ekman (Neumann, \& Lozo, 2012); 2) pruebas informatizadas que utilizan imágenes GIF en las que se presentan imágenes con rostros neutrales y otras con rostros que expresan distintos estados emocionales (Kohler, Bilker, Hagendoorn, Gur, \& Gur, 2000); 3) pruebas que utilizan imágenes esquemáticas de los rostros expresando emociones (Palermo, Pasqualetti, Barbati, Intelligente, \& Rossini, 2006).

Gran parte de la importancia que poseen las pruebas de reconocimiento de expresiones faciales de emociones radica en que permiten evaluar a sujetos con diferentes patologías para determinar su grado de habilidad en el reconocimiento de emociones (Bediou et al., 2012). De este modo, existen estudios que tratan de determinar el grado de deterioro que puede causar la enfermedad en el reconocimiento de expresiones faciales emocionales, por ejemplo, en personas con autismo (Corbett et al., 2009), demencias (Kessels et al., 2007; Maki, Yoshida, Yamaguchi, \& Yamaguchi, 2013), esquizofrenia (Cavieres y Valdebenito, 2007; Chen, Cataldo, Norton, \& Ongur, 2012) y fundamentalmente depresión en adultos (Anderson et al., 2011) y niños (López-Duran, Kuhlman, George, \& Kovacs, 2012).

Sin embargo, a pesar del valor que poseen estos instrumentos necesarios para el reconocimiento de emociones faciales, no existen pruebas conformadas con fotografías de personas del medio local argentino, por lo cual se hace sumamente necesaria la construcción de pruebas adaptadas a las características de esta población. El primer paso para ello lo constituye la realización de un análisis bibliométrico sobre investigaciones científicas que utilicen pruebas de reconocimiento de emociones faciales. De este modo, los resultados obtenidos en cuanto a la valoración de sus alcances, limitaciones y las pruebas mayormente utilizadas, podrían ser utilizados como base para la construcción de una prueba local de reconocimiento de emociones faciales. Por lo tanto, el presente trabajo tiene la finalidad de realizar un estudio bibliométrico de artículos científicos publicados entre 1976 y agosto de 2011 que utilicen pruebas de reconocimiento de emociones faciales.

\section{Método}

\section{Muestra}

Para el presente estudio la muestra fue obtenida por medio de la consulta de bases de datos de texto completo pertenecientes a literatura científica de psicología, neuropsicología, psiquiatría y neurología, tanto nacionales como internacionales (esto incluyó literatura en español, inglés y portugués).

En los casos en que se contaba únicamente con los resúmenes fueron contactados los autores para acceder al artículo completo por vía e-mail. Las bases de datos de texto completo consultadas fueron: LILACS, Redalyc, Scielo, Dialnet, PsycINFO (APA), ScienceDirect y Scopus (Elsevier), Interscience (Wiley/Balckwell), WilsonWeb (Wilson), SpringerLink (Springer) y EBSCO. 


\section{Criterios de inclusión y exclusión de la bibliografía consultada}

La búsqueda de artículos científicos abarcó el período de tiempo comprendido entre los años 1976 y agosto de 2011. Esto se decidió en función que las fotografías del POFA fueron publicadas en 1976 por Paul Ekman, el cual ha servido como plataforma para el desarrollo de pruebas posteriores, por lo tanto, y de acuerdo a la ley de crecimiento exponencial de la literatura científica, se espera que la productividad en investigaciones que utilicen pruebas de reconocimiento de emociones faciales aumente progresivamente a través del tiempo (Arenas y Romero, 2003).

Se trabajó con artículos completos publicados en revistas científicas en inglés, español y portugués. Quedaron excluidos de la muestra: estudios teóricos, informes de congresos, tesis y aquellos artículos los cuales se halló un solapamiento entre las diversas bases de datos, así como investigaciones que no tuvieran referencias teóricas en las cuales sustentarse ni explicitaran el autor del mismo. La decisión de utilizar artículos científicos publicados en revistas de prestigio se fundamentó en que éstos, para ser divulgados, han sido sometidos a un exhaustivo proceso de evaluación realizado por expertos (Russell, 2004).

\section{Términos de búsqueda}

Para realizar la búsqueda de trabajos científicos pertinentes a este estudio se utilizaron como guía los siguientes descriptores:

En español: reconocimiento de emociones faciales, emociones, pruebas de reconocimiento de emociones, expresiones faciales.

En portugués: reconhecimento de emoções faciais, emoções, prova de reconbecimento de emocões, expressões faciais.

En inglés: facial emotions rocognition, emotions, emotions recognition test, facial expressions.

\section{Procedimiento}

Se utilizó un diseño ex post facto retrospectivo historiográfico bibliométrico de acuerdo a la clasificación de Montero y León (2002), aún cuando la misma conserve muchas de las características de lo que los mismos autores habían denominado anteriormente estudios descriptivos mediante análisis de documentos. En este diseño la población de estudio corresponde a revistas publicadas en un período de tiempo pasado y el análisis de datos se apoya en datos cuantitativos bibliométricos, sin excluir interpretaciones cualitativas (Montero y León, 2002).

La realización de una investigación bibliométrica supone tres momentos: localización y selección de documentación, revisión de la información documental y análisis de los datos documentales (Rosa, Huertas y Blanco, 1996).

En la primera etapa se realizó la búsqueda de artículos según los criterios establecidos en la muestra. En la segunda etapa se ordenó la información obtenida de acuerdo a los indicadores bibliométricos. Una vez clasificada la información, se procedió al análisis de los datos documentales, lo que constituye el tercer momento de un estudio bibliométrico. Para este análisis se emplearon indicadores bibliométricos de productividad y análisis de materia.

\section{Índice $\mathbf{H}$}

$\mathrm{El}$ índice $\mathrm{H}$ fue publicado por Jorge Hirsch (Hirsch, 2005), ocasionando controvertidas reacciones. El mismo mide la calidad profesional en función de la cantidad de citas que han tenido las investigaciones dentro de la comunidad científica que trata el tema de estudio (Costas y Bordons, 2007). El propio Hirsch no deja de reconocer las limitaciones que tiene el índice $\mathrm{H}$ : no mide la importancia ni el impacto que tienen los trabajos y se limita a revelar la calidad o relevancia profesional que tiene un investigador en una determinada área de estudio en relación a otros profesionales en un definido período de tiempo (Salgado y Páez, 2007).

En el presente trabajo el Índice H se extrajo a través del buscador Harzing's Publish or Perish versión 3.4.4351 (Harzing, 2007) el cual proporciona información acerca de documentos e investigaciones en línea; presenta recursos para la evaluación de la investigación y la calidad de las revistas, así como el software para realizar análisis de citas.

\section{Análisis de datos}

Una vez obtenidos los indicadores bibliométricos de productividad y análisis de materia, se realizó un análisis de datos utilizando Tablas de distribución de frecuencias, en donde se incluyó información descriptiva tales como porcentajes y frecuencias. Para facilitar la apreciación de los datos se utilizaron diferentes representaciones gráficas.

\section{Resultados}

Como resultado de la búsqueda bibliográfica en las distintas bases de datos se hallaron 465 trabajos científicos en el período comprendido entre 1976 y agosto de 2011, teniendo en cuenta los criterios de inclusión y exclusión establecidos previamente.

\section{Productividad}

Este indicador permitió determinar la evolución temporal de la producción científica existente sobre pruebas de reconocimiento de emociones faciales, así como la productividad de autores, instituciones, revistas y países.

\section{Evolución temporal de la productividad}

En función de los resultados obtenidos se determinó que el primer artículo publicado sobre el tema fue en el año 1978, siendo la única publicación existente en el lustro de 1975. Es a partir del año 1995 cuando la producción co- 
mienza a aumentar, siendo el lustro más productivo el 2005 con un total de 204 trabajos. A su vez, pudo apreciarse una diferencia importante entre la productividad de los últimos tres lustros ya que comprenden un $90.96 \%$ de la producción en relación con los lustros comprendidos entre 1975 y 1995 , los cuales reportaron un total de $7.96 \%$ de los trabajos publicados.
$\mathrm{Al}$ analizar la evolución temporal por año de la productividad (Figura 1) se reflejó un crecimiento sistemático siendo cada vez más pronunciado a partir del año 1995, mientras que en los años comprendidos entre 1978 y 1994 sólo hubo una publicación por año, a excepción de los años comprendidos entre 1979-1987 y 1990.

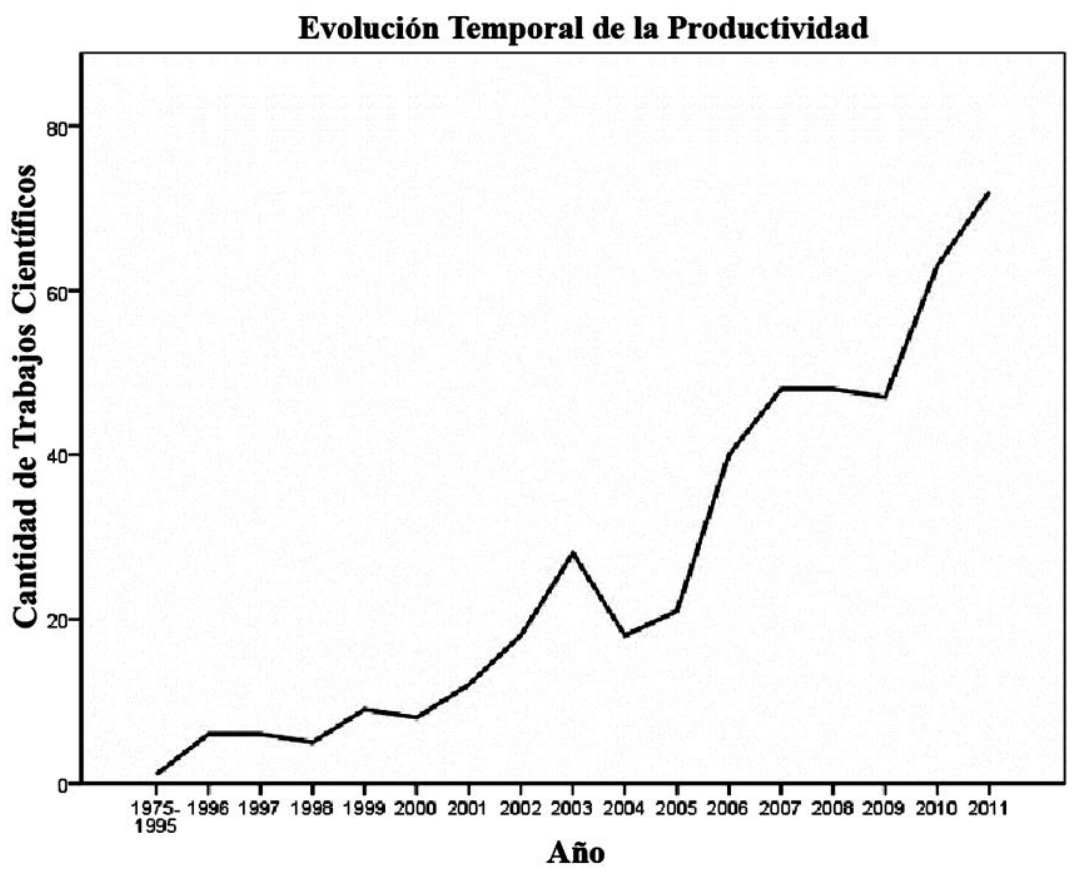

Figura 1. Evolución temporal por año de la productividad. Período: enero, 1976 - agosto, 2011. Se agrupó el intervalo 1975-1995 ya que comprenden sólo el 7.96\% del total de documentos.

\section{Productividad de autores}

A partir de los 465 trabajos científicos analizados se identificaron los autores que investigan sobre pruebas de reconocimiento de emociones faciales, para lo cual, en primer lugar, se registró la cantidad de autores existentes en la totalidad de documentos y en segundo lugar, se computó la cantidad de veces que cada uno de los autores firmó un trabajo.
De esta manera, con la información recabada se elaboró una tabla de frecuencias absolutas y relativas que permitió determinar la productividad de autores.

De este modo, en la Tabla 1 se observa que, sobre el total de 1637 autores, el $82.59 \%$ firmaron solamente 1 trabajo, mientras que sólo un $0.06 \%$ aporta 17 firmas. Luego, se visualiza que un $11.67 \%$ de los autores tienen 2 firmas, y un $2.81 \%$ de los autores efectuaron 3 firmas.

Tabla 1. Distribución de autores según su número de firmas.

\begin{tabular}{|c|c|c|c|c|}
\hline Autores con N Firmas & Cantidad de Autores & $\%$ de Autores & Cantidad Total de Firmas & Porcentaje de Firmas \\
\hline Autores con 17 firmas & 1 & 0.06 & 17 & 0.79 \\
\hline Autores con 13 firmas & 1 & 0.06 & 13 & 0.61 \\
\hline Autores con 12 firmas & 1 & 0.06 & 12 & 0.56 \\
\hline Autores con 9 firmas & 2 & 0.12 & 18 & 0.84 \\
\hline Autores con 7 firmas & 4 & 0.24 & 28 & 1.31 \\
\hline Autores con 6 firmas & 9 & 0.55 & 54 & 2.52 \\
\hline Autores con 5 firmas & 8 & 0.49 & 40 & 1.87 \\
\hline Autores con 4 firmas & 22 & 1.34 & 88 & 4.11 \\
\hline Autores con 3 firmas & 46 & 2.81 & 138 & 6.44 \\
\hline Autores con 2 firmas & 191 & 11.67 & 382 & 17.83 \\
\hline Autores con 1 firmas & 1352 & 82.59 & 1352 & 63.12 \\
\hline TOTAL & 1637 & 100 & 2142 & 100 \\
\hline
\end{tabular}


En cuanto a la productividad de los autores según la cantidad de firmas, se registró un total de 2142 firmas. Como se puede observar en la Tabla 1, la mayoría de los autores (1352 autores) realizaron 1 firma, representando el $63.12 \%$ del total. A su vez, 191 autores firmaron 2 trabajos, dando lugar a 382 firmas $(17.83 \%)$, mientras que 46 autores firmaron 3 trabajos, aportando con esto 138 firmas (6.44\%). Por último, también se observó un autor con 17 firmas $(0.79 \%)$, uno con 13 firmas $(0.61 \%)$ y otro con 12 firmas $(0.56 \%)$.

\section{Autores más productivos}

Con la información recabada se identificaron los 9 autores más productivos del total de la muestra, los cuales son: Gur Ruben, C. con 17 trabajos, aportando el 3.66\% del total de éstas; seguido por Calder Andrew, J. con 13 trabajos, reflejando el 2.80\%; Young Andrew, W. con 12 estudios, dando lugar al 2.58\%; luego siguen Kohler Christian, G. y Perrett David, I. con 9 trabajos, representando el 1.94\% cada uno. Los últimos cuatro autores realizan 7 trabajos cada uno, registrando el $1.51 \%$ del total de los trabajos. Estos datos son presentados en la Tabla 2.

En cuanto a la afiliación de los autores más productivos, 3 de ellos pertenecen a University of Pennsylvania, siendo ésta la universidad más productiva del total de la muestra.

\section{Índice $\mathrm{h}$}

El índice h permitió valorar la calidad profesional a través de la cantidad de investigaciones y calidad de las mismas a partir de la tasa de citas. Dicho índice sólo fue aplicado a los autores más productivos. Para obtenerlo se utilizó el programa Harzing's (Harzing, 2007) en el cual se incluyeron todas las disciplinas. Los resultados revelan que Young Andrew, W. presenta el índice más alto. A continuación se encuentra Gur Ruben, C. y Gur Raquel, E., siendo Derntl Birgit el autor con el menor índice $\mathrm{h}$ (Tabla 2).

Debido a que este indicador se calcula a partir de toda la bibliografía a la que se puede acceder desde Google Schoolar $\mathrm{y}$, por ende, incluye la variedad de temas que trabaja cada autor, se decidió realizar un cálculo paralelo utilizando sólo aquellos trabajos que estuvieran relacionadas al reconocimiento de emociones faciales, lo cual se aprecia en la última columna de la Tabla 2.

\section{Productividad de instituciones}

La productividad institucional fue medida a través del número de trabajos científicos. La misma se expuso en una tabla de distribución de frecuencias en la cual se detalló el número de trabajos realizados por cada institución (Tabla 3). Los resultados mostraron un total de 551 instituciones con 1025 trabajos, de las cuales sólo 1 institución cuenta con 24 trabajos, mientras que 372 instituciones aportaron 1 solo trabajo.

Se observó que, del total de instituciones, el $95.47 \%$ posee entre 1 y 5 trabajos, concentrando un $77.45 \%$ del total de los documentos, a su vez, sólo el 4.53\% de las instituciones tiene entre 6 y 24 trabajos, dando lugar a un $22.55 \%$ de las investigaciones.

Tabla 2. Autores más productivos, su afiliación e Índice h.

\begin{tabular}{|c|c|c|c|c|}
\hline Autor & $\mathrm{N}^{\circ}$ de Trabajos & Afiliación & Índice $\mathrm{h}$ & $\begin{array}{l}\text { Índice } \mathrm{h} \text { en relación al tema del recono- } \\
\text { cimiento de emociones faciales }\end{array}$ \\
\hline Gur Ruben, C. & 17 & University of Pennsylvania & 83 & $\begin{array}{ll}27 \\
\end{array}$ \\
\hline Calder Andrew, J. & 13 & University of Cambridge & 45 & 21 \\
\hline Young Andrew, W. & 12 & MRC Applied Psychology Unit & 120 & 19 \\
\hline Kohler Christian, G. & 9 & University of Pennsylvania & 36 & 14 \\
\hline Perrett David, I. & 9 & University of St Andrews & 54 & 7 \\
\hline Derntl Birgit & 7 & $\begin{array}{l}\text { University of Aachen Medical University of } \\
\text { Vienna University of Vienna }\end{array}$ & 11 & 8 \\
\hline Gur Raquel, E. & 7 & University of Pennsylvania & 76 & 18 \\
\hline Harmer Catherine, J. & 7 & University of Oxford & 27 & 13 \\
\hline Kryspin-Exner Ilse & 7 & University of Vienna & 13 & 10 \\
\hline
\end{tabular}

Tabla 3. Distribución de instituciones según el número de trabajos científicos.

\begin{tabular}{|c|c|c|c|c|}
\hline Instituciones con N Trabajos & $\mathrm{N}^{\circ}$ de Instituciones & $\%$ de Instituciones & $\mathrm{N}^{\circ}$ de Trabajos & $\%$ de Trabajos \\
\hline Instituciones con 24 trabajos & 1 & 0.18 & 24 & 2.34 \\
\hline Instituciones con 15 trabajos & 1 & 0.18 & 15 & 1.46 \\
\hline Instituciones con 14 trabajos & 1 & 0.18 & 14 & 1.37 \\
\hline Instituciones con 11 trabajos & 4 & 0.73 & 44 & 4.29 \\
\hline Instituciones con 9 trabajos & 4 & 0.73 & 36 & 3.51 \\
\hline Instituciones con 8 trabajos & 5 & 0.91 & 40 & 3.90 \\
\hline Instituciones con 7 trabajos & 4 & 0.73 & 28 & 2.73 \\
\hline Instituciones con 6 trabajos & 5 & 0.91 & 30 & 2.93 \\
\hline Instituciones con 5 trabajos & 13 & 2.36 & 65 & 6.34 \\
\hline Instituciones con 4 trabajos & 18 & 3.27 & 72 & 7.02 \\
\hline Instituciones con 3 trabajos & 39 & 7.08 & 117 & 11.41 \\
\hline Instituciones con 2 trabajos & 84 & 15.25 & 168 & 16.39 \\
\hline Instituciones con 1 trabajos & 372 & 67.51 & 372 & 36.29 \\
\hline Total & 551 & 100 & 1025 & 100 \\
\hline
\end{tabular}




\section{Instituciones más productivas según número de trabajos científicos}

En la Tabla 4 se detallan las 16 instituciones más productivas teniendo en cuenta el número de trabajos que cada una de ellas aportó. La institución más productiva es University of Pennsylvania con 24 documentos, le sigue University of California con 15 trabajos y University of St Andrews con 14 artículos. Luego, como puede observarse, el número de trabajos disminuye sistemáticamente.

\section{Productividad de revistas}

A partir de los datos recabados, se identificó un total de 160 revistas en inglés, español y portugués. Se calculó la productividad de las revistas en función de la cantidad de trabajos publicados. En la Tabla 5 se puede observar que sólo una revista cuenta con 63 documentos, mientras que la mayor proporción de revistas cuenta con sólo 1 publicación. Las restantes revistas se distribuyeron de forma decreciente en función del número de trabajos publicados.

\section{Revistas más productivas según número de trabajos científicos}

Del total compuesto por 160 revistas con el que se trabajó, se seleccionaron 15 que se destacaron por ser las más productivas y se agruparon en la Tabla 6.

Tabla 4. Las 16 instituciones más productivas según el número de trabajos científicos que cada una aporta.

\begin{tabular}{lc}
\hline Instituciones & No de Trabajos $^{\text {o }}$ \\
\hline University of Pennsylvania & 24 \\
University of California & 15 \\
University of St Andrews & 14 \\
Harvard Medical School, Massachusetts General Hospi- & 11 \\
tal & 11 \\
University College London & 11 \\
University of Düsseldorf & 11 \\
University of York & 9 \\
Institute of Psychiatry & 9 \\
MRC Cognition and Brain Sciences Unit & 9 \\
University of Newcastle & 9 \\
University of Vienna & 8 \\
Kyoto University & 8 \\
National Institute of Mental Health & 8 \\
University of Hong Kong & 8 \\
University of New South Wales & 8 \\
University of Sydney & \\
\hline
\end{tabular}

Tabla 5. Distribución de revistas según número de trabajos científicos. El número total de trabajos que figura aquí (461) no se corresponde con el número total de documentos analizados ya que, existen 4 artículos $(0,86 \%)$ que no informan sobre la revista de publicación.

\begin{tabular}{|c|c|c|c|c|}
\hline Revistas con N Trabajos & Cantidad de Revistas & \% de Revistas & $\mathrm{N}^{\circ}$ de Trabajos & $\%$ de Trabajos \\
\hline Revistas con 63 Trabajos & 1 & 0.63 & 63 & 13.55 \\
\hline Revistas con 48 Trabajos & 1 & 0.63 & 48 & 10.32 \\
\hline Revistas con 28 Trabajos & 1 & 0.63 & 28 & 6.02 \\
\hline Revistas con 12 Trabajos & 1 & 0.63 & 12 & 2.58 \\
\hline Revistas con 11 Trabajos & 1 & 0.63 & 11 & 2.37 \\
\hline Revistas con 9 Trabajos & 2 & 1.25 & 18 & 3.87 \\
\hline Revistas con 8 Trabajos & 2 & 1.25 & 16 & 3.44 \\
\hline Revistas con 7 Trabajos & 3 & 1.88 & 21 & 4.52 \\
\hline Revistas con 6 Trabajos & 3 & 1.88 & 18 & 3.87 \\
\hline Revistas con 5 Trabajos & 4 & 2.50 & 20 & 4.30 \\
\hline Revistas con 4 Trabajos & 5 & 3.13 & 20 & 4.30 \\
\hline Revistas con 3 Trabajos & 12 & 7.50 & 36 & 7.74 \\
\hline Revistas con 2 Trabajos & 26 & 16.25 & 52 & 11.18 \\
\hline Revistas con 1 Trabajo & 98 & 61.25 & 98 & 21.08 \\
\hline TOTAL & 160 & 100 & 461 & 99.14 \\
\hline
\end{tabular}

Tabla 6. Revistas más productivas según número de trabajos científicos.

\begin{tabular}{|c|c|c|c|}
\hline Revista & $\mathrm{N}^{\circ}$ de Trabajos & $\%$ de Trabajos & País de edición \\
\hline Neuropsychologia & 63 & 13.55 & Reino Unido \\
\hline Psychiatry Research & 48 & 10.32 & Países Bajos \\
\hline Schizophrenia Research & 28 & 6.02 & Países Bajos \\
\hline Brain and Cognition & 12 & 2.58 & Estados Unidos \\
\hline Cortex & 11 & 2.37 & Italia \\
\hline Personality and Individual Differences & 9 & 1.94 & Canadá \\
\hline NeuroImage & 9 & 1.94 & Estados Unidos \\
\hline Journal of Autism and Development Disorders & 8 & 1.72 & Estados Unidos \\
\hline International Journal of Psychophysiology & 8 & 1.72 & Países Bajos \\
\hline The Spanish Joumal of Psychology & 7 & 1.51 & España \\
\hline Psychopharmacology & 7 & 1.51 & Alemania \\
\hline Journal of Affective Disorders & 7 & 1.51 & Países Bajos \\
\hline Research in Developmental Disabilities & 6 & 1.29 & Estados Unidos \\
\hline Neuroscience Letters & 6 & 1.29 & Países Bajos \\
\hline Emotion & 6 & 1.29 & Estados Unidos \\
\hline
\end{tabular}


Se observa que Neuropsychologia es la revista más productiva ya que cuenta con 63 artículos, le sigue Psychiatry Research con un total de 48 trabajos. Estas dos revistas contienen un número significativo de trabajos publicados relacionados al tema de referencia, las restantes fueron distribuidas de manera decreciente, realizando un corte que permitiera agrupar únicamente aquellas que contaran con más de 6 artículos en su haber.

\section{Posibilidad de acceso a las revistas}

Se determinó que de un total de 160 revistas con las que se trabajó, un $88 \%$ son de acceso restringido, lo que implica la necesidad de una suscripción paga o de la compra de los artículos para su acceso, mientras que un $12 \%$ de éstas son de acceso libre y gratuito (“Open Access").

Entre estas últimas, se pueden mencionar algunas como: "Journal of Psychiatry and Neuroscience", "BMC Neuroscience", "Behavioral and Brain Functions", "Psicothema", "Psicologica", "Comunicar", "Revista Chilena de NeuroPsiquiatría".

\section{Productividad de países}

Para determinar los países que se destacaron por su productividad (distribución de trabajos por país), se consideró la afiliación institucional de cada autor $\mathrm{y}$, de este modo, se agruparon los trabajos según país de origen como se muestra en la Tabla 7. Así, se determinó que Estados Unidos es el país más productivo, ya que cuenta con 141 documentos. Le sigue Reino Unido, que tiene en su haber un total de 83 documentos y dejó para Alemania el tercer lugar con 62 documentos. En función del análisis realizado, cabe resaltar el lugar en el que quedó ubicada Argentina, ya que sólo cuenta 3 trabajos.

\section{Colaboración entre autores}

Con el fin de evaluar la colaboración entre autores se elaboró la Tabla 8 en la que se muestra, en la primera columna, la cantidad de firmas que aparecen por artículo, en la segunda se incluyó el número de trabajos científicos que se corresponden con los valores ubicados en la primera columna, le sigue la columna en la que se plasmaron los valores porcentuales que conciernen a las frecuencias absolutas ubicadas en la segunda columna. Finalmente, en las dos columnas restantes, se ubicaron los valores que representan el número de firmas, el cual se obtuvo multiplicando el total de firmas por el número de los trabajos, y el porcentaje correspondiente.
Tabla 7. Distribución de los trabajos según país de origen.

\begin{tabular}{|c|c|c|}
\hline País & $\mathrm{N}^{\circ}$ de Trabajos & $\%$ \\
\hline Estados Unidos & 141 & 23.58 \\
\hline Reino Unido & 85 & 14.21 \\
\hline Alemania & 62 & 10.37 \\
\hline Australia & 30 & 5.02 \\
\hline España & 28 & 4.68 \\
\hline Canadá & 27 & 4.52 \\
\hline Japón & 27 & 4.35 \\
\hline Italia & 26 & 4.35 \\
\hline Francia & 25 & 4.18 \\
\hline Países Bajos & 17 & 2.84 \\
\hline Austria & 16 & 2.68 \\
\hline Bélgica & 11 & 1.84 \\
\hline China & 11 & 1.84 \\
\hline Brasil & 9 & 1.51 \\
\hline Finlandia & 8 & 1.34 \\
\hline Colombia & 7 & 1.17 \\
\hline India & 7 & 1.17 \\
\hline Suiza & 7 & 1.17 \\
\hline Israel & 5 & 0.84 \\
\hline Hungría & 4 & 0.67 \\
\hline Nueva Zelanda & 4 & 0.67 \\
\hline Turquía & 4 & 0.67 \\
\hline Rusia & 4 & 0.67 \\
\hline Argentina & 3 & 0.50 \\
\hline Hong Kong & 3 & 0.50 \\
\hline Corea del Sur & 3 & 0.50 \\
\hline Chile & 2 & 0.33 \\
\hline Cuba & 2 & 0.33 \\
\hline Grecia & 2 & 0.33 \\
\hline Irlanda & 2 & 0.33 \\
\hline México & 2 & 0.33 \\
\hline Singapur & 2 & 0.33 \\
\hline Sur África & 2 & 0.33 \\
\hline No especifica & 2 & 0.33 \\
\hline Bosnia Herzegovina & 1 & 0.17 \\
\hline Corea & 1 & 0.17 \\
\hline Escocia & 1 & 0.17 \\
\hline Holanda & 1 & 0.17 \\
\hline Polonia & 1 & 0.17 \\
\hline Portugal & 1 & 0.17 \\
\hline Suecia & 1 & 0.17 \\
\hline Tailandia & 1 & 0.17 \\
\hline TOTAL & 598 & 100 \\
\hline
\end{tabular}

De este modo, si se toma como ejemplo la segunda fila de la Tabla, se observa que se analizaron 79 trabajos que cuentan con 2 firmas cada una, lo que muestra que un $16.99 \%$ de los documentos se encuentran firmados por dos autores, esto a su vez da un total de 158 firmas que representan un $7.38 \%$ del total. 
Tabla 8. Distribución de los trabajos según número de firmas.

\begin{tabular}{lcccc}
\hline Firmas & $\begin{array}{c}\mathrm{N}^{\circ} \mathrm{de} \\
\text { Trabajos }\end{array}$ & \% de Trabajos & $\begin{array}{c}\mathrm{N}^{\circ} \text { de Fir- } \\
\text { mas }\end{array}$ & $\begin{array}{c}\% \text { de Fir- } \\
\text { mas }\end{array}$ \\
\hline 1 & 11 & 2.37 & 11 & 0.51 \\
2 & 79 & 16.99 & 158 & 7.38 \\
3 & 75 & 16.13 & 225 & 10.50 \\
4 & 93 & 20.00 & 372 & 17.37 \\
5 & 75 & 16.13 & 375 & 17.51 \\
6 & 44 & 9.46 & 264 & 12.32 \\
7 & 35 & 7.53 & 245 & 11.44 \\
8 & 20 & 4.30 & 160 & 7.47 \\
9 & 14 & 3.01 & 126 & 5.88 \\
10 & 7 & 1.51 & 70 & 3.27 \\
11 & 8 & 1.72 & 88 & 4.11 \\
12 & 4 & 0.86 & 48 & 2.24 \\
\hline TOTAL & 465 & 100 & 2142 & 100 \\
\hline
\end{tabular}

A partir del análisis de estos datos se rescató que sólo 11 trabajos fueron firmados por un único autor, siendo los restantes 454 producidos en coautoría. La mayor parte de los documentos han sido firmados por 2 y 4 autores. Finalmente, se observó que un escaso número de los estudios fueron firmados por 10 autores o más.

\section{Índice de Colaboración}

En función de los datos expresados en la Tabla presentada anteriormente (Tabla 8), se calculó el índice de colaboración. Éste se obtuvo dividiendo la cantidad total de las firmas por el total de trabajos examinados.

$$
\text { Índice de Colaboración (I.C) = Firmas / Trabajos }
$$

$$
\text { I.C }=4.61
$$

En función de dicha operación, se obtuvo un índice de colaboración de 4,61, computado a partir de 2142 firmas sobre 465 trabajos. Este resultado muestra que, en promedio los documentos cuentan con 4 firmas o más cada uno.

\section{Análisis de materia}

Dentro de este indicador se consideró la población estudiada, el diseño metodológico empleado, los instrumentos de evaluación utilizados y los objetivos perseguidos en cada investigación.

\section{Población}

Para conocer el tipo de población con la que se trabajó en las investigaciones científicas, se realizaron Tablas de distribución de estudios según la población estudiada. De este modo, dicha población se clasificó, en primer lugar, según la edad y, en segundo lugar, a partir del género.

\section{Tipo de población según la edad}

En efecto, para clasificar a la población según la edad, se establecieron las siguientes categorías:

- Niños: de 0 a 11 años.

- Adolescentes: de 12 a 21 años.

- Adultos: de 22 a 64 años.

- Adultos mayores: de 65 años en adelante.

El 68.62\% (387 estudios) utilizaron población adulta. En segundo lugar se encuentra la población adolescente, la cual aparece en 74 estudios, representando un $13.12 \%$ del total. Luego, con una proporción relativamente similar, se encuentran los niños y los adultos mayores, poblaciones identificadas en $50(8.87 \%)$ y $47(8.33 \%)$ estudios, respectivamente. Por último, se clasificó como "No Especifica" a aquellos estudios que no informaron sobre la edad de la población estudiada (6 estudios; $1.06 \%$ ).

Tipo de población según el género

Además se clasificó a la población estudiada en función del género y se sumó la categoría "Mixto", en donde se incluyeron aquellos trabajos científicos que utilizaron población de ambos géneros. Por otra parte, en la categoría "No Especifica", se agruparon los estudios que no informaron sobre el género de los sujetos de investigación.

La gran mayoría de las investigaciones trabajaron con sujetos de ambos géneros (Categoría: Mixto), representando un $87.31 \%$ del total de documentos analizados. Mientras que fueron pocas las que emplearon población de un sólo género, ya fuera femenino o masculino, esto es un $6.24 \%$ y un $5.38 \%$, respectivamente. Por otro lado, sólo un 1.08\% (5 artículos) no informaron sobre el género de la población estudiada.

\section{Diseños metodológicos}

Con el propósito de identificar los diseños metodológicos o tipos de estudios desarrollados por las investigaciones científicas que utilizan pruebas de reconocimiento de emociones faciales, se empleó la clasificación de León y Montero (2005). Debido a que los estudios teóricos se excluyeron de este trabajo, las categorías quedaron conformadas del siguiente modo:

1 - Estudios descriptivos mediante observación.

2 - Estudios descriptivos mediante encuestas.

3 - Estudios cualitativos.

4 - Estudios experimentales.

5 - Estudios cuasi-experimentales.

6 - Estudios ex post facto.

7 - Estudios experimentales de caso único.

8 - Estudios instrumentales. 
No se encontraron estudios descriptivos ni cualitativos y los estudios ex post facto fueron los más numerosos ya que del total de trabajos científicos, $288(61.94 \%)$ son de este tipo. En segundo lugar, se ubicaron los estudios experimentales, los cuales fueron desarrollados por 127 investigaciones científicas $(27.31 \%)$. Por último, con una proporción similar, aparecen los diseños cuasi-experimentales (18 artículos), experimentales de caso único (16 artículos) e instrumentales (16 artículos), representando el primero de éstos, un 3.87\% del total de artículos y, los dos restantes, un 3.44\% cada uno.

\section{Instrumentos de evaluación}

Con el propósito de analizar los instrumentos utilizados en las investigaciones científicas, se tuvieron en cuenta tanto las pruebas de reconocimiento de emociones faciales propiamente dichas, así como también aquellos instrumentos diseñados con otra finalidad pero utilizados en el estudio para evaluar la habilidad de reconocer emociones faciales. Del total de los trabajos científicos analizados, en 414 se utilizó un sólo instrumento de evaluación representando un $89.03 \%$.

El primer lugar, los instrumentos fueron clasificados según consistieran en:

- Fotografías de personas expresando emociones,

- Imágenes GIF,

- Esquemas,

- Videos.

Según se revela en la Tabla 9, la proporción de estudios que utilizaron fotografías como instrumentos de evaluación es significativamente mayor: 485 estudios. 23 estudios emplearon videos, 16 estudios esquemas y 2 utilizaron imágenes GIF.

Tabla 9. Distribución de estudios según el/los tipo/s de instrumento/s de evaluación que utilicen. ${ }^{*}$ El número total de estudios (526) no se corresponde con el número total de trabajos analizados (465) ya que en algunos documentos se realizó más de un estudio y el $11 \%$ de las investigaciones utiliza más de un instrumento.

\begin{tabular}{lcc}
\hline Tipo de Instrumento & $\mathrm{N}^{\circ}$ de Estudios* & \% de Estudios \\
\hline Fotografías & 485 & 92.21 \\
Imágenes GIF & 2 & 0.38 \\
Esquemas & 16 & 3.04 \\
Videos & 23 & 4.37 \\
\hline Total & 526 & 100 \\
\hline
\end{tabular}

Por otra parte, la Tabla 10 muestra cuáles fueron los instrumentos más utilizados por las investigaciones científicas. Como se puede apreciar, si bien las fotografías obtenidas por Ekman en 1976 no constituyen un instrumento de evaluación, si se han utilizado como tal en 168 estudios. En segundo lugar se encuentra el Japanese and Caucasian Facial Expressions of Emotion (JACFEE), empleado en 27 estudios. Por último, siguen con una proporción marcadamente inferior, Nimstim Face y Karolinska Directed Emotional Face (11 estudios), Japanese and Caucasian Neutral Faces (JAC-
NeuF), International Affective Picture System (IAPS), Facial Expressions of Emotion: Stimuli and Tests (FEEST) y Emotion Hexagon Test. El resto de los estudios que emplearon otros instrumentos fueron agrupados en la categoría "Otros".

Tabla 10. Instrumentos más utilizados por los estudios científicos. *El número total de estudios (526) es diferente del número total de trabajos (465) ya que en algunos documentos se realizaron varios estudios.

\begin{tabular}{lcc}
\hline Nombre del Instrumento & $\mathrm{N}^{\circ}$ de Estudios* & \% de Estudios \\
\hline POFA & 168 & 31.94 \\
JACFEE & 27 & 5.13 \\
NIMSTIN FACE & 11 & 2.09 \\
KAROLINSKA DIRECTED & 11 & 2.09 \\
EMOTIONAL FACES & 10 & 1.90 \\
JACNEUF & 10 & 1.90 \\
IAPS & 10 & 1.90 \\
FEEST & 10 & 1.90 \\
EMOTION HEXAGON & 269 & 51.14 \\
TEST & 526 & 100 \\
OTROS & & \\
Total & &
\end{tabular}

\section{Evolución temporal de los instrumentos}

Como se observa en la Tabla 11, en la mayoría de las investigaciones se utilizó el POFA, apareciendo por primera vez en el lustro de 1985. Según muestran las frecuencias absolutas, su empleo aumentó a través de los lustros, desde 2 a 70 documentos (en los lustros 1985 y 2005, respectivamente). Pero, si se tienen en cuenta las frecuencias relativas, se observa que esta prueba experimenta una disminución sistemática de su empleo en relación a los otros instrumentos. En efecto, disminuyó desde un 100\% (utilizado en los úni$\cos 2$ trabajos del lustro de 1985) hasta un 25,66\% (empleado en 39 trabajos en el lustro de 2010).

Tabla 11. Evolución temporal por lustro de los instrumentos empleados en los trabajos científicos. *En el lustro de 1975 no fue estudiado el año 1975, mientras que en el lustro de 2010 sólo fueron estudiados los años 2010 y 2011

\begin{tabular}{lcccc}
\hline Lustro & POFA & $\%$ & OTROS & $\%$ \\
\hline $1975^{*}$ & - & - & 1 & 100 \\
1980 & - & - & - & - \\
1985 & 2 & 100 & - & - \\
1990 & 4 & 80 & 1 & 20 \\
1995 & 16 & 51.61 & 15 & 48.39 \\
2000 & 36 & 38.30 & 58 & 61.70 \\
2005 & 70 & 30.04 & 163 & 69.96 \\
$2010^{*}$ & 39 & 25.66 & 113 & 74.34 \\
\hline No Especifica & 1 & 12.50 & 7 & 87.50 \\
\hline
\end{tabular}

Los demás instrumentos tuvieron una aparición más tardía que el POFA y su empleo presentó un marcado crecimiento a través de los lustros. Es decir, tanto si se tienen en cuenta las frecuencias absolutas (desde 1 a 113 trabajos), como las relativas (desde $20 \%$ a $74.34 \%$ ), la utilización de otros instrumentos crece en relación al empleo del POFA.

La Figura 1 y la Tabla 12 establecen una comparación entre la evolución temporal de la utilización del POFA y la 
evolución temporal del empleo del resto de los instrumentos de evaluación. De este modo, se puede observar que hasta 1998 tanto el POFA como los demás instrumentos se utilizaron relativamente poco, alcanzando valores similares. A partir aquí, comenzaron a tomar ventaja los demás instrumentos, al mismo tiempo que el POFA decrece en su empleo en el año 2000. En el 2001 tuvo lugar una pequeña ven-

Tabla 12. Distribución por año de los estudios científicos según los instrumentos empleados.

\begin{tabular}{lccccc}
\hline Año & POFA & $\%$ & Otros & $\%$ & \\
\hline $1976-1977$ & - & - & - & - & \\
1978 & - & - & 1 & 100 & 1 \\
$1979-1987$ & - & - & - & - & \\
1988 & 1 & 100 & - & - & 1 \\
1989 & 1 & 100 & - & - & 1 \\
1990 & - & - & - & - & \\
1991 & 1 & 100 & - & - & 1 \\
1992 & 1 & 100 & - & - & 1 \\
1993 & 1 & 50 & 1 & 50 & 2 \\
1994 & 1 & 100 & - & - & 1 \\
1995 & 2 & 50 & 2 & 50 & 4 \\
1996 & 4 & 66.67 & 2 & 33.33 & 6 \\
1997 & 4 & 57.14 & 3 & 42.86 & 7 \\
1998 & 3 & 60 & 2 & 40 & 5 \\
1999 & 3 & 33.33 & 6 & 66.67 & 9 \\
\hline
\end{tabular}

taja a favor del empleo del POFA y, desde este punto, tanto la utilización del POFA como del resto de los instrumentos comenzó a experimentar un crecimiento pronunciado y sistemático. Pero a pesar del crecimiento de la utilización de todos los instrumentos, la ventaja se incrementa cada vez más a favor del resto de los instrumentos en detrimento del POFA.

\begin{tabular}{lccccc}
\hline Año & POFA & $\%$ & Otros & $\%$ & \\
\hline 2000 & 2 & 22.22 & 7 & 77.78 & 9 \\
2001 & 7 & 53.85 & 6 & 46.15 & 13 \\
2002 & 7 & 35 & 13 & 65 & 20 \\
2003 & 11 & 35.48 & 20 & 64.52 & 31 \\
2004 & 9 & 42.86 & 12 & 57.14 & 21 \\
2005 & 7 & 31.82 & 15 & 68.18 & 22 \\
2006 & 15 & 31.91 & 32 & 68.09 & 47 \\
2007 & 14 & 24.56 & 43 & 75.44 & 57 \\
2008 & 21 & 37.50 & 35 & 62.50 & 56 \\
2009 & 13 & 25.49 & 38 & 74.51 & 51 \\
2010 & 16 & 21.92 & 57 & 78.08 & 73 \\
2011 & 23 & 29.11 & 56 & 70.89 & 79 \\
NO ESPECIFICA & 1 & 12.50 & 7 & 87.50 & 8 \\
\hline TOTAL & 168 & 31.94 & 358 & 68.06 & 526 \\
\hline
\end{tabular}

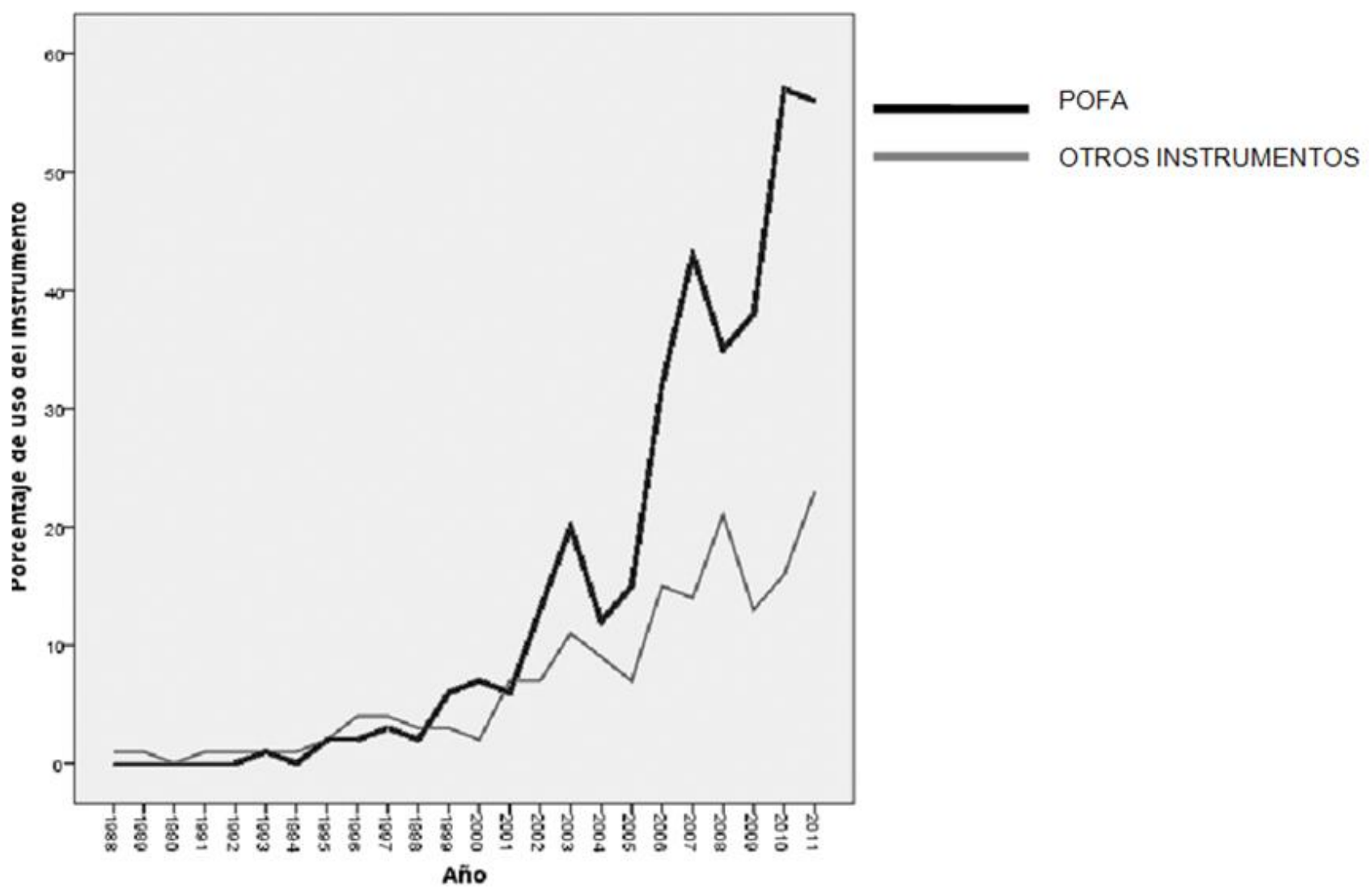

Figura 2. Evolución temporal por año de los instrumentos utilizados en los trabajos científicos, desde 1988 hasta agosto de 2011. 


\section{Objetivos de las investigaciones científicas}

Con la finalidad de analizar con qué objetivo cada investigación utilizó una prueba de reconocimiento de emociones faciales, se realizó la Figura 3 que distribuye los estudios según su objetivo fuera estudiar una patología, algún aspecto de la normalidad o realizar un desarrollo científicotecnológico.

Como se observa en el Gráfico, la gran mayoría de los estudios (298 estudios; 63.81\%) tuvieron como objeto de análisis alguna patología. A su vez, 157 estudios (33.62\%) evaluaron algún aspecto de la normalidad y, con una proporción significativamente menor (2.57\%) 12 estudios se enfocaron en el desarrollo científico-tecnológico.

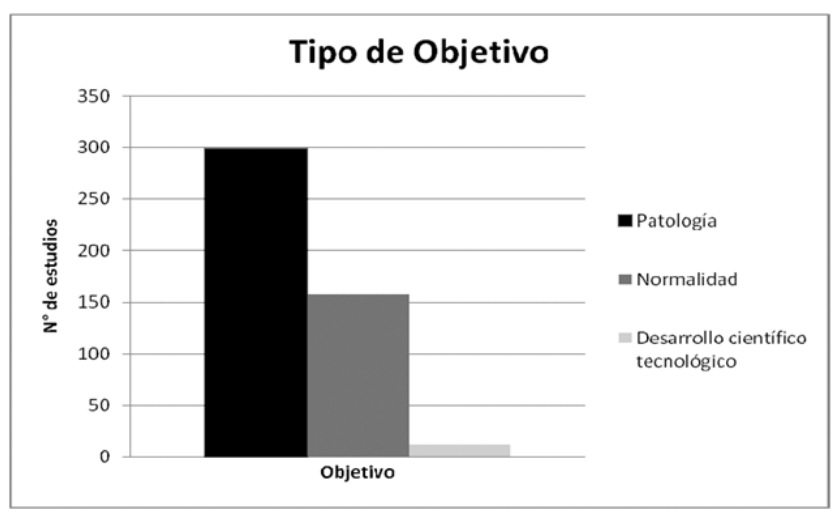

Figura 3. Objetivos estudiados por los trabajos científicos.

Asimismo, también se efectuó un análisis para determinar cuáles fueron las patologías más frecuentemente estudiadas en relación al tema del reconocimiento de emociones faciales. Los resultados obtenidos se expresan en la Tabla 13. Como se aprecia en la misma, las patologías más estudiadas fueron la Esquizofrenia y Demencias, mientras que las menos estudiadas han sido la Psicopatía, Trastornos Alimentarios y Abuso Sexual del niño y del adulto.

Tabla 13. Distribución de los estudios según el tipo de objetivo estudiado.

\begin{tabular}{lcc}
\hline Patología & $\mathrm{N}^{\circ}$ de Estudios & $\%$ de Estudios \\
\hline Esquizofrenia & 75 & 25.17 \\
Demencias & 61 & 20.47 \\
Lesiones Cerebrales & 39 & 13.09 \\
Trastornos del Neurodesarrollo & 37 & 12.42 \\
Trastornos del Estado de Animo & 34 & 11.41 \\
Consumo de Sustancias & 20 & 6.71 \\
Otros Trastornos Psiquiátricos & 19 & 6.40 \\
Psicopatía & 6 & 2.01 \\
Trastornos Alimentarios & 4 & 1.34 \\
Abuso Sexual de Niño/Adulto & 3 & 1.01 \\
\hline
\end{tabular}

\section{Discusión}

El presente trabajo consiste en un estudio bibliométrico sobre investigaciones científicas publicadas entre 1976 y agosto de 2011 que utilizan pruebas de reconocimiento de emocio- nes faciales. En este sentido, la finalidad de este trabajo es brindar información sobre el estado actual de la ciencia en relación a las pruebas de reconocimiento de emociones faciales que contribuya a la posterior construcción de un instrumento local destinado a medir dicha habilidad.

En efecto, este análisis bibliométrico se realizó en base a una muestra de 465 artículos científicos publicados en diferentes revistas, según los criterios de inclusión y exclusión detallados oportunamente. Cabe aclarar que esta selección implica un sesgo de publicación debido a que sólo se trabajó con artículos publicados y es posible que existan trabajos relacionados a este tema que aún no hayan sido publicados. En relación a esto, Russell (2004) sostiene que, si bien los estudios bibliométricos permiten conocer el estado actual de la producción científica sobre determinada temática, los datos pueden no reflejar en forma totalmente fiel el "estado actual de la cuestión", justamente debido a que muchos artículos pueden encontrarse en proceso de revisión o en prensa.

Entre los resultados obtenidos se puede destacar el indicador de productividad, el cual permitió estimar la evolución temporal del proceso de producción en el período comprendido entre 1976 y agosto de 2011. Los datos indican que el primer artículo publicado sobre pruebas de reconocimiento de emociones faciales fue en el año 1978. Es en los últimos tres lustros cuando la productividad comienza a aumentar de manera sistemática, siendo el 2005 el lustro más productivo con un total de 204 documentos. Respecto al lustro actual resultaría impropio realizar juicios sobre la producción científica y su supuesto crecimiento ya que sólo se tuvieron en cuenta los años 2010 y 2011.

La progresión observada en la productividad a través del tiempo permite corroborar la ley de crecimiento exponencial postulada por Price (1963), la cual afirma que el crecimiento de la información científica se duplica cada 10 o 15 años. Una de las causas del creciente desarrollo de la producción científica podría deberse al aumento del interés en las cuestiones relacionadas a las emociones faciales y a la consiguiente revalorización de las pruebas que evalúan dicha habilidad por su contribución al diagnóstico de ciertas patologías.

En cuanto a la productividad de autores se obtuvo un total de 1637 investigadores con 2142 firmas. Los datos reflejan que la mayoría de los autores firmaron una sola publicación, mientras que una minoría de ellos tienen 5 o más firmas. Estos resultados concuerdan con la ley de dispersión propuesta por Bradford, la cual afirma que la información sobre un determinado tema se distribuye en zonas concéntricas de productividad decreciente. Aun así, el hecho que los autores se destaquen por firmar una mayor cantidad de investigaciones no significa que tengan mayor impacto ni mayor relevancia dentro de la comunidad científica. Es por esto que se recurrió al Índice $h$ para medir la calidad de los investigadores en base a la relación que existe entre el número de trabajos científicos y el número de citas que se hacen de éstas. Los resultados muestran que los autores que presentan una mayor calidad profesional según el índice h no son los mismos que presentan mayor producción de investigaciones. 
Cabe aclarar que el programa Harzing's Publish or Perish con el que se obtiene el índice h presenta algunas limitaciones: en primer lugar sólo analiza la bibliografía accesible mediante el motor de búsqueda Google Schoolar, por lo cual deja fuera una vasta cantidad de producciones que no se encuentran en este buscador. Y también, puede que existan autores homónimos transfiriéndose ese sesgo a dicho índice. De este modo, las limitaciones mencionadas anteriormente hacen que el índice $\mathrm{h}$ obtenido a través de dicho programa no refleje fielmente la calidad de cada investigador analizado, por lo cual resultaría inapropiado extraer conclusiones de tales resultados. Todo esto constituye una razón para la posterior realización de un estudio bibliométrico que incluya un método alternativo para calcular la calidad de los investigadores, que supere las deficiencias del programa Harzing's, y permita la obtención de valores más representativos de dicho indicador.

Con respecto a las instituciones, se obtuvo un total de 551 instituciones con 1025 documentos. Un dato interesante es que el autor más productivo, Gur Ruben, C. pertenece a la University of Pennsylvania, la cual es la Universidad con mayor productividad. Esto permite estimar la importancia que se le otorga a las investigaciones sobre pruebas de reconocimiento de emociones faciales en esta institución en comparación con las demás instituciones.

Por otro lado, con respecto a la productividad de revistas, se determinó que, de un total de 160 revistas, las más productivas son Neuropsychologia, Psychiatry Research y Schizophrenia Research. Además, se identificaron 19 revistas de libre acceso u "Open Access", que representan sólo un 12\% del total, dejando en evidencia que el acceso al conocimiento científico de un modo libre y gratuito aún resulta muy limitado. Con respecto a la productividad de países, se estableció el orden jerárquico de éstos en función de la cantidad de trabajos publicados. En este sentido, se destacaron con un amplio margen de diferencia respecto al resto Estados Unidos, Reino Unido y Alemania. Esto último resulta de suma importancia para el objetivo del presente trabajo, debido a que Argentina sólo tiene en su haber un total de 3 documentos referidos al tema, lo cual invita a reflexionar sobre la necesidad de investigar acerca del reconocimiento de emociones faciales en este país.

En cuanto a la coautoría de los trabajos, se efectuó el cálculo del indicador de colaboración entre los autores de los trabajos científicos. De este modo, a partir del número total de firmas y el número de trabajos analizados, el índice de colaboración resultó ser de 4,61. Esto implica que, en promedio, los artículos cuentan con 4 firmas o más cada uno. Se puede inferir que esto tiene su causa en el desarrollo y crecimiento de las redes de información en los últimos años, en los que ya no ha sido necesaria la copresencia para realizar aportes a una investigación, dando lugar al trabajo en equipo de autores que se ubican en diferentes países. Así, tal como sostiene Lopez-Lopez (1996), a mayor grado de colaboración corresponde mayor grado de especulación, lo que indica la evolución de la ciencia hacia lo empírico y experimental.
En relación al tipo de población utilizada en las investigaciones, se observa que los adultos constituyen el segmento más estudiado en el área. En segundo lugar se encuentra el empleo de la población adolescente, mientras que las poblaciones menos estudiadas son los niños y los adultos mayores. Esto denota la importancia de comenzar a investigar, por un lado, con población infantil de modo tal que los descubrimientos sirvan de base para diagnosticar enfermedades de manera temprana y detectar problemas en la interacción social. Por otro lado, también resulta importante realizar investigaciones con adultos mayores sobre indicadores conductuales de la emoción que permitan realizar mejoras en su calidad de vida.

En relación a los diseños metodológicos se destacaron los estudios ex post facto y experimentales. Ambos son de tipo empírico, y suelen ser el tipo de artículos que se publican usualmente en revistas, mientras que los desarrollos teóricos tienden a exponerse en libros (Del Prette \& Del Prette, 2002). Los estudios teóricos no están incluidos en el presente trabajo, por lo tanto, esto constituye una razón para incluir en un futuro estudio bibliométrico, otros tipos de documentos como ser libros, estudios de maestría y doctorado, disertaciones en congresos, entre otros.

Otra explicación probable al hecho que, en la mayoría de los casos analizados se utilicen estudios ex post facto puede deberse a que en las investigaciones la población más estudiada presenta algún tipo de patología, por lo cual no todas las variables pueden ser manipuladas, es decir, tal como sostiene Kerlinger (1982), en este tipo de estudios no se puede tener control directo sobre las variables independientes porque ya acontecieron sus manifestaciones o por ser intrínsecamente manipulables.

Con respecto a los instrumentos utilizados en los estudios científicos, cabe destacar que el $92.21 \%$ de éstos consisten en fotografías. Por su parte, el Picture Of Facial Affect se utilizó como prueba de reconocimiento de emociones faciales en la mayoría de los estudios, seguido por instrumentos que se emplean en una proporción marcadamente inferior. Si se tiene en cuenta la evolución temporal del empleo de instrumentos, se puede observar que la implementación tanto del POFA como del resto de los instrumentos crece significativamente a través de los años, pero a partir de 1999 la utilización de los demás instrumentos se incrementa cada vez más en detrimento del empleo del POFA. Esta desventaja que experimenta la utilización del POFA en relación a la de los demás instrumentos, se podría explicar por el hecho que, a medida que aumenta el interés y, por tanto, la investigación en el campo de las emociones faciales, van construyéndose cada vez más instrumentos que permiten evaluar la habilidad para el reconocimiento de tales emociones y, de este modo, se incrementa de manera muy pronunciada la cantidad de instrumentos diferentes del POFA. A su vez, la gran proliferación de este tipo de herramientas en los últimos años, puede derivarse de la necesidad de contar con instrumentos actualizados y adaptados a la población bajo estudio, por lo cual el POFA, creado en 1976 en EEUU, resulta 
inapropiado para estudios más actuales y/o en otros contextos socio-culturales.

En relación a los objetivos perseguidos por las diversas investigaciones se observó que a lo largo de los años se mantuvo constante el análisis de la esquizofrenia en relación al reconocimiento de emociones faciales, al igual que el estudio de los distintos tipos de demencias. Se debe realizar la salvedad que la primera investigación que se llevó a cabo sobre dicho tema tuvo como objetivo el estudio de los trastornos del neurodesarrollo. En función de lo expuesto anteriormente, se puede detectar la necesidad de realizar investigaciones que tengan en cuenta otros objetivos como por ejemplo, la relación entre el reconocimiento de emociones faciales y distintos tipos de discapacidad ya que éste resultó ser uno de los campos menos estudiados.

Como se mencionó oportunamente, este análisis bibliométrico presenta ciertas limitaciones. Una de ellas es el llamado sesgo de publicación, el cual implica la imposibilidad de contar con la totalidad de los trabajos existentes sobre la temática estudiada. Por otra parte, una de las dificultades encontrada en la construcción de indicadores bibliométricos fue la dificultad para identificar algunos datos bibliográficos debido a la falta de criterios unificados tanto en las bases de datos como en las revistas. Esto concuerda con lo sostenido por Russell (2004), donde la falta de un nivel profundo de normalización de los datos en los documentos fuentes constituye un problema. De todos modos, se debe tener en cuenta que los dos tipos de limitaciones mencionados anteriormente son propios de los estudios bibliométricos (De Filippo y Fernandez, 2002; Spinak, 1998). A su vez, existen otras limitaciones que pueden considerarse como precursoras de nuevas investigaciones que intenten superarlas, logrando resultados más exhaustivos y, por ende, más representativos.

\section{Referencias}

Adolphs, R. (2003). Cognitive neuroscience of human social behavior. Nature Reviews Neuroscience, 4, 165-178.

Anderson, I. M., Shippen, C., Juhasz, G., Chase, D., Thomas, E., Downey, D., et al. (2011). State-dependent alteration in face emotion recognition in depression. The British Journal of Psychiatry, 198, 302-308.

Arenas, S. y Romero, A. (2003). Indicadores Bibliométricos de la Revista Científica Zootecnia Tropical. Zootecnia Tropical, 21, 325-350.

Bediou, B., Brunelin, J., d'Amato, T., Fecteau, S., Saoud, M., Hénaff, M. A., \& Krolak-Salmon, P. (2012). A comparison of facial emotion processing in neurological and psychiatric conditions. Frontiers in Psychology, 3:98. Doi: 10.3389/fpsyg.2012.00098.

Cavieres, A. y Valdebenito, M. (2007). Déficit en el reconocimiento de emociones faciales en la esquizofrenia. Implicancias clínicas y neuropsicológicas. Revista Chilena de Neuropsiquiatría, 45, 120-128.

Chen, Y., Cataldo, A., Norton, D. J., \& Ongur, D. (2012). Distinct facial processing in schizophrenia and schizoaffective disorders. Schizophrenia Research, 134, 95-100.

Corbett, B. A., Carmean, V., Ravizza, S., Wendelken, C., Henry, M. L., Carter, C. et al. (2009). A functional and structural study of emotion and face processing in children with autism. Psychiatry Research, 173, 196205.

Costas, R., Bordons, M. (2007), The h-index: advantages, limitations and its relation with other bibliometric indicators at the micro-level. Journal of Informetrics, 1(3), 193-203.
Dichas limitaciones son por ejemplo el no haber evaluado la colaboración entre autores también desde su estructura social (detección de grupos de colaboración o colegios invisibles) y el no considerar a los estudios teóricos dentro de la muestra ni haber consultado fuentes bibliográficas como ser libros, informes de congresos y tesis.

Para concluir, se puede afirmar que, a pesar de todas las limitaciones, el presente análisis bibliométrico constituye un trabajo de suma importancia ya que contribuye al conocimiento de la actividad científica referida a las pruebas de reconocimiento de emociones faciales, sistematizando datos relacionados a este tema, evaluándolos cuantitativamente e identificando las tendencias actuales en este campo de estudio. Este proceso de sistematización del conocimiento científico sirve como base para la posterior construcción de un instrumento adaptado a la población argentina que evalúe la habilidad para el reconocimiento de emociones faciales de una manera válida y confiable, constituyendo esto un gran aporte tanto para la investigación como para la evaluación clínica.

En todo este recorrido resulta evidente cómo el proceso científico posibilita un acercamiento entre la clínica y la investigación, dónde el quehacer del psicólogo se va construyendo tanto en la actividad científica, a través de la investigación de campos de conocimientos, la construcción de instrumentos y la evaluación de la ciencia, como así también en la labor clínica, a partir de la utilización de tales instrumentos como herramientas al servicio del diagnóstico y tratamiento de patologías.

Agradecimientos.- Este estudio ha sido subsidiado por la Secretaría de Ciencia y Tecnología de la Universidad Nacional de Córdoba.

De Filippo, D. y Fernández, M. T. (2002). Bibliometría: importancia de los indicadores bibliométricos. Documento recuperado de ricyt.org/index.php.

Del Prette, Z. y Del Prette, A. (2002). Psicología de las habilidades sociales. Terapia y educación. México: Manual Moderno.

Ekman, P. y Friesen, W.V. (1976). Pictures of Facial Affect. Palo Alto, California: Consulting Psychology Press.

Ekman, P. y Friesen, W.V. (1978). Facial action coding system: A technique for the measurement of facial movement. Palo Alto, Calif.: Consulting Psychologists Press.

Ekman, P. (1972). Universals and cultural differences in facial expression of emotion. Nebraska symposium on motivation, 1971. Universidad de Nebraska. Documento recuperado de http://www.paulekman.com/wpcontent/uploads/2009/02/Emotional -And-Conversational-Nonverbal-Signals.pdf

Ekman, P. (2003). Emotions Revealed. Nueva York: Times Books.

Ekman, P., Sorenson, E. R. y Friesen, W.V. (1969). Pan-cultural elements in facial displays of emotion. Science, 164, 86-88.

Hamm, J., Kohler, C. G., Gur, R. C., \& Verma, R. (2011). Automated Facial Action Coding System for dynamic analysis of facial expressions in neuropsychiatric disorders. Journal of Neuroscience Methods, 200, 237-256.

Harzing, A. W. (2007). Publish or Perish. Recuperado de http://www.harzing.com/pop.htm

Herba, C. y Phillips, M. (2004). Annotation: development of facial expression recognition from childhood to adolescence: behavioral an neuro- 
logical perspectives. Journal of Child Psychology and Psychiatry and Allied Disciplines, 45, 1185-1198.

Hirsch, J. E. (2005). An index to quantify an individual's scientific research output. Proccedings of the National Academy of Sciences of the United States of America, 102, 16569-16572.

Iglesias, J., Loeches, A. y Serrano, J. (1989). Expresión facial y reconocimiento de emociones en lactantes. Infancia y Aprendizaje, 48, 93-113.

Izard, C. E. (1971). The face of emotion. New York: Appleton-Century-Crofts.

Kerlinger, F. (1982). Investigación del comportamiento. Técnicas y metodología. México: Editorial Interamericana.

Kessels, R. P., Gerritsen, L., Montagne, B., Ackl, N., Diehl, J. y Danek, A. (2007). Recognition of facial expressions of different emotional intensities in patients with frontotemporal lobar degeneration. Behavioural Neurology, 18, 31-36.

Kohler, C. G., Bilker, W., Hagendoorn, M., Gur, R. E., \& Gur, R. C. (2000). Emotion recognition deficit in schizophrenia: association with symptomatology and cognition. Biological Psychiatry, 48, 127-136.

León, O. y Montero, I. (2005). Sistema de clasificación del método en los informes de investigación en Psicología. International Journal of Clinical and Health Psychology, 5, 115-127.

Lopez-Duran, N. L., Kuhlman, K. R., George, C., \& Kovacs, M. (2012). Facial emotion expression recognition by children at familial risk for depression: high-risk boys are oversensitive to sadness. Journal of Child Psychology and Psychiatry, Doi: 10.1111/jcpp.12005.

Maki, Y., Yoshida, H., Yamaguchi, T., \& Yamaguchi, H. (2013). Relative preservation of the recognition of positive facial expression "happiness" in Alzheimer disease. International Psychogeriatrics, 25, 105-110.

Matsumoto, D., Keltner, D., Shiota, M.N., O'Sullivan, M. y Frank, M. (2008). Facial expression of emotions. En Lewis, M., Haviland- Jones, J. y Barrett, L. (Eds.), Handbook of emotions. New York: Guilford Press.

Montero, I. y León, O. G. (2002). Clasificación y descripción de las metodologías de investigación en Psicología. Revista Internacional de Psicología Clinica y de la Salud/International Journal of Clinical and Health Psychology, 2, 503-508.

Neumann, R., \& Lozo, L. (2012). Priming the activation of fear and disgust: evidence for semantic processing. Emotion, 12, 223-228.
Palermo, M. T., Pasqualetti, P., Barbati, G., Intelligente, F., \& Rossini, P.M. (2006). Recognition of schematic facial displays of emotion in parents of children with autism. Autism: The International Journal of Research and Practice, 10, 353-364.

Porter, S., ten Brinke, L., Baker, A., \& Wallace, B. (2012). Would I lie to you? "Leakage" in deceptive facial expressions relates to psychopathy and emotional intelligence. Personality and Individual Differences, 51, 133137.

Porter, S., ten Brinke, L., \& Wallace, B. (2012). Secrets and lies: Involuntary leakage in deceptive facial expressions as a function of emotional intensity. Journal of Non-verbal Behaviour, 36, 23-37.

Preuschoft, S. (2000). Primate faces and facial expressions. Social Research, 67, 245-271.

Price, D. J. S. 1963. Little science, big science. New York: Columbia University Pres.

Rosa, A., Huertas, J. A. y Blanco, F. (1996). Metodología de la bistoria de la psicología. Madrid: Editorial Alianza.

Russell, J. M (2004). Obtención de Indicadores Bibliométricos a Partir de la Utilización de las Herramientas Tradicionales de Información. Documento recuperado de http://www.eventos.bvsalud.org.

Salgado, J. F. y Páez, D. (2007). La productividad científica y el índice H de Hirchs de la psicología social Española: convergencia entre indicadores de productividad y comparación con otras áreas. Psicothema, 19, 179-189.

Spinak, E. (1998). Indicadores cienciométricos. Ciencias de la Información, 27 (2), 141-148.

Sutcliffe, A., Dunbar, R., Binder, J., \& Arrow, H. (2012). Relationships and the social brain: integrating psychological and evolutionary perspectives. British Journal of Psychology, 103, 149-168.

Ten Brinke, L., Macdonald, S., Porter, S., \& O'Connor, B. (2012). Crocodile tears: facial, verbal and body language behaviours associated with genuine and fabricated remorse. Law and Human Behavior, 36, 51-59.

Wong, N., Beidel, D. C., Sarver, D. E., \& Sims, V. (2012). Facial emotion recognition in children with high functioning autism and children with social phobia. Child Psychiatry and Human Development, 43, 775-794.

(Articulo recibido: 15-9-2012; revisado: 15-2-2012; aceptado: 18-3-2013) 